\title{
Gesundheitszentren als Antwort auf die Zukunft oder gefährliche Konkurrenz?
}

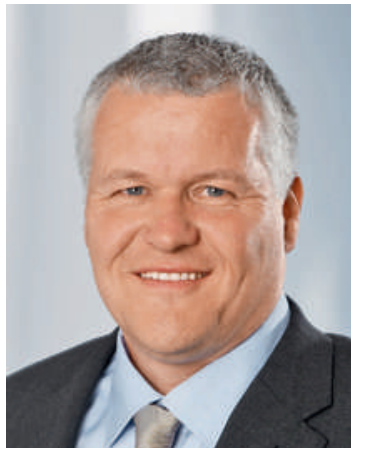

Der Begriff «Gesundheitszentrum» begegnet uns in jüngster Zeit regelmässig, sei es als Ausdruck für Qualitätsstandards im Betreuungsprozess der Patientinnen und Patienten oder als Bezeichnung für die ambulante multidisziplinäre Zusammenarbeit. Auch der Blick in die Kantone zeigt, dass der Begriff unterschiedlich verstanden wird. Der Regierungsrat des Kantons Bern hat im Jahr 2011 in seiner Antwort auf eine Motion vier Begriffe zur Charakterisierung von Gesundheitszentren aufgeführt: Notfallversorgung, tagesklinische Behandlung, Assessment/Triage und SpezialistenSprechstunden, wobei der Allgemeinmediziner als zentraler Spezialist auftritt.

Grundsätzlich kann man sagen, dass sich ein Gesundheitszentrum entweder als rein ärztliche Institution präsentiert oder als Gruppe ärztlicher und paramedizinischer Fachpersonen. Im Vordergrund steht die Vernetzung der Betreuung in Form interdisziplinärer Zusammenarbeit. Minimalanforderungen bestehen nicht, ein Gesundheitszentrum hat sich jedoch regionalen und situativen Bedürfnissen anzupassen. Damit ein Gesundheitszentrum effizient funktioniert, muss auch seine finanzielle und therapeutische Unabhängigkeit garantiert sein.

\section{Im Vordergrund steht die Vernetzung der Betreuung in Form interdiszipli- närer Zusammenarbeit.}

Bei Gesundheitszentren, welche in Spitäler mit öffentlichem Leistungsauftrag integriert sind, kommt es zu einer Wettbewerbsverzerrung, zum Beispiel durch die Abgeltung der gemeinwirtschaftlichen Leistungen als versteckte Subvention solcher Institutionen. Treten Gemeinden als Betreiber von Gesundheitszentren auf, so investieren sie Steuergelder in Zentren, die eigentlich anderswo benötigt würden. Bei privaten Investoren und Versicherern wiederum führen wirtschaftliche Interessen zu Interessenskonflikten.

In Frankreich zum Beispiel werden Gesundheitszentren von staatlicher Seite gefördert und durch die Krankenversicherungen unterstützt: Zum einen werden entsprechende politische und rechtliche Rahmenbedingungen geschaffen, zum anderen ist die finanzielle Unterstützung in Form von Krediten möglich, sofern die Kriterien erfüllt sind. Ein solches Kriterium ist beispielsweise das Bereitstellen von Notfallzimmern, womit die Zentren einen wesentlichen Beitrag zur Notfallversorgung im Rahmen der Basisversorgung leisten. Als Grundsatz gilt für Gesundheitszentren in Frankreich, dass sie im therapeutischen Entscheidungsprozess unabhängig sind. Schliesslich soll ein Gesundheitszentrum einen Mehrwert für den Patienten wie auch für das Fachpersonal und die Verantwortlichen der Gesundheitsversorgung, sprich die Kantone, bieten. Dieser besteht aber nicht alleine in Form von Kostenoptimierung, sondern auch im Sinne eines breiten Angebots an Leistungen verschiedenster Akteure des Gesundheitswesens. Der Informationsfluss zwischen den Akteuren der Medizin, Pflege, Pharmazie, Physiotherapie usw. wird optimiert, verkürzt und vereinfacht, wenn sich die verschiedenen Akteure regelmässig vor Ort austauschen. Therapien werden diskutiert und wenn nötig Algorithmen erstellt. Diese dienen einerseits allen Fachper-

\section{Wenn die Kantone den Gesundheits- zentren klare Versorgungsaufträge übertragen möchten, sollten hierzu die Spielregeln genau definiert werden.}

sonen, die in den Behandlungsprozess involviert sind, und stellen anderseits ein wichtiges Arbeitsinstrument dar, um Behandlungsfehler zu reduzieren. Selbstverständlich gibt es bereits diverse Formen von Qualitätszirkeln, aber oft beschränken sich diese auf Ärztinnen und Ärzte und beziehen das paramedizinische Fachpersonal nicht mit ein.

Wir müssen uns bewusst sein, dass Gesundheitszentren kein Garant dafür sind, die Kosten zu reduzieren. Insbesondere bevölkerungsschwache Regionen profitieren zwar von Gesundheitszentren als Attraktionsförderern, gleichzeitig kann sich aber bei zyklisch ungünstigem Nachfrage-AngebotsVerhältnis die einzelne Leistung auch deutlich verteuern.

Der Begriff «Gesundheitszentrum» beschränkt sich weder auf den ambulanten Sektor der Spitäler noch ausschliesslich auf die privaten Institutionen. Aber wenn die Kantone den Gesundheitszentren mehr Kompetenz und klare Versorgungsaufträge übertragen möchten, sollten hierzu die Spielregeln genau definiert werden. Das heisst, dass insbesondere was die Subventionen, Taxpunktwerte und Finanzierungsmodalitäten betrifft, verbindliche Rahmenbedingungen geschaffen werden müssen, um keine Beliebigkeit zu fördern. Und trotz ökonomischer Aspekte stehen immer die Patientinnen und Patienten bzw. die Bürger im Zentrum des ärztlichen Handelns - das wollen wir nicht vergessen!

Dr. med. Remo Osterwalder, Mitglied des Zentralvorstandes der FMH, Departementsverantwortlicher Freipraktizierende Ärztinnen und Ärzte 